\title{
Computational Modeling of FRP Reinforced Cementitious Beams
}

\author{
Sonia Marfia-Elio Sacco \\ Dipartimento di Meccanica, Strutture, A. T., Università di Cassino, Cassino, Italy
}

In the present paper, a one-dimensional elastoplastic-damage model for the analysis of the mechanical response of beams constituted by cementitious materials, i.e., concrete or masonry, strengthened by fiber reinforced polymers (FRP), is developed. The analysis is performed for a typical section, representing an elementary part of beam characterized by the finite length, defined as the distance between two fractures. A thermodynamically consistent model is proposed; it takes into account the different behavior in tension and in compression of the cohesive materials.

The governing equations are derived and a numerical procedure is developed. It is based on the arc-length method, within an implicit Euler algorithm for the time integration. An accurate choice of the control parameters is performed. The finite step nonlinear problem is solved adopting a Newton-Raphson scheme within a predictorcorrector procedure.

Some numerical examples are developed in order to analyze the non trivial axial and bending behavior of reinforced concrete and masonry beams and to assess the efficiency of the proposed procedure. Comparisons with analytical solutions are reported.

\section{INTRODUCTION}

In the last decade, great interest has been devoted by the technological and the scientific community to the possibility of using advanced composites materials, such as fiber reinforced polymers (FRP), to repair and reinforce concrete and masonry elements [1-4].

In fact, these materials present a combination of excellent properties, such as low weight, immunity to corrosion, possibility of formation in very long lengths and high mechanical strength and stiffness. They can be successfully applied to the tensile zones of structural members by using epoxy adhesives. Moreover, FRP materials are very simple to install (resulting in low labor costs) and they are also removable, which is a very interesting property mainly for monumental structures.

For all these reasons, the use of FRP for strengthening civil structures is becoming very popular and several experimental

Received 30 October 2004; accepted 10 January 2006.

Address correspondence to Sonia Marfia-Elio Sacco, Dipartimento di Mecconica, Strutture A. \& T., Via Di Biasio 43, Cassino 03043, Italy. E-mail: sacco@unicas.it and theoretical investigations have been developed to evaluate the effectiveness of the application of FRP and to define rules for the design of the reinforcement.

Several studies related to the behavior of concrete beams, reinforced by FRP sheets, have been developed. Among the others, Rabinovitch and Frostig [5] developed a nonlinear analysis of the response of cracked reinforced concrete beams, retrofitted by externally bonded FRP strips. Nonlinear constitutive relations for the various materials, equilibrium and deformation compatibility are taken into account in the model. The nonlinear implicit equations governing the problem are solved through an iterative procedure. Alagusundaramoorthy et al. [6] and Deniaud and Chen [7] derived the shear and bending response of FRPreinforced elements. Rabinovitch [8] proposed an analytical investigation of the bending behavior of reinforced concrete beams strengthened by composite materials externally bonded using nonlinear and inelastic adhesives. The model is derived through the virtual work taking into account the compatibility conditions and the constitutive laws. The numerical results show that the use of inelastic and nonlinear adhesives improves the overall load carrying behavior of strengthened beams increasing ductility and reducing the shear stress near the ends of the bonded strips. Shao et al. [9] presented a nonlinear inelastic analysis for large displacement of the cyclic response of concrete columns reinforced with FRP, developing a three node composite beam-column finite element. The constitutive models for cyclic loading of FRP and concrete are taken into account.

FRP materials are even adopted to reinforce masonry structures, such as walls, arches and vaults. Several studies have been devoted to the analysis of masonry panels. Experimental investigations were developed by Schwegler [10], Ehsani [11] and Laursen et al. [12] in order to evaluate the mechanical response of reinforced masonry walls subjected to seismic action. Tumialan et al. [13] emphasized the positive aesthetics and social impact related to the use of FRP to strengthen masonry walls against in-plane and out-of-plane collapse.

An analysis of masonry arch reinforced by FRP sheets was proposed by Luciano et al. [14], developing an experimental, as well as a finite element study. Foraboschi [15] presented a mathematical model able to predict the ultimate load associated 
with the possible failure modes of reinforced arches. Marfia and Sacco [16] developed a finite element model based on no-tension material with limited strength in compression to study the structural collapse of masonry elements reinforced by FRP materials. It is noted that the failure of reinforced structures is often due to the limited strength in compression of the masonry, so that the inelastic behavior of the masonry in compression should be taken into account for a correct design of the reinforcement and, mainly, for an accurate determination of the failure load.

Micromechanical models able to derive the response of reinforced masonry have been developed, considering the masonry as a composite material with the blocks as inclusions in a matrix of mortar [17-19].

It can be stated that the knowledge on the use of FRP in civil constructions and on the design for the reinforcement of existing structures can be considered quite advanced, so that some guidelines are today available with the aim to help the engineers properly design strengthening interventions. The last and maybe more complete guideline, including also the case of masonry structures, has been developed in Italy [20].

It is fair to notice that the studies regarding the application of FRP materials are mainly developed for retrofitting, strengthening and wrapping existing concrete and masonry structural members in order to increase the bending capacity and shearing resistance. In fact, the application of FRP in these structural members leads to a significant increase of the structural stiffness and strength; but it can also induce a very tough and brittle mechanical response of reinforced element, because of the brittle or quasi-brittle behavior of the cementitious and FRP materials [21]. Moreover, it has been demonstrated that the tension-stiffening effect for cementitious elements strengthened by FRP — plate/sheets is very pronounced. In fact, it has been shown that the presence of a quite low amount of external reinforcement is able to provide a significant reduction of axial deformability in cracked elements within a strong reduction of width of transverse cracks and of the distance between cracks [22].

Because of the reduced crack distance and crack opening, continuous damage models can be successfully adopted for the analysis of cementitiuos, concrete or masonry, beams. In fact, the so-called fiber models [9, 23, 24], based on damage mechanics and plasticity, can be used to investigate the mechanical behavior of cementitious beams reinforced by FRP materials. In particular, the concrete, as well as the masonry can be modeled considering a brittle behavior in traction and inelastic deformations accompanied by damage effects in compression [25].

The aim of this paper is the definition of a simple and effective structural model to investigate on the behavior of cementitious (concrete or masonry) beams, reinforced by advanced composite laminates glued on the top and bottom of the beam. In particular, the specific objective of the work is the determination and investigation of the possible very complex equilibrium paths resulting from the softening response of the concrete or masonry material.
To this end, a one-dimensional thermodynamically consistent model for cementitious materials, which takes into account the damage and the plasticity effects is proposed. It is assumed that the plasticity can be activated only when the material is subjected to a compressive stress; on the contrary, the material behavior in tension is governed only by damage. The constitutive equations as well as the damage and plasticity evolutive equations are written in explicit form. The damage and plastic evolutions result coupled since the damage is governed by the elastic strain.

Because of the damage in tension and the damage and plasticity in compression, highly nonlinear equilibrium equations are deduced. In order to solve the nonlinear problem, a numerical procedure is developed. It is based on the arc-length method, with a proper choice of the control parameters. The evolutionary equations of the damage and plasticity are integrated with respect to the time, developing an implicit Euler algorithm. The finite step nonlinear solution is obtained performing a predictor-corrector procedure. In particular, the coupled damage and plasticity evolutive equations are solved adopting a Newton-Raphson algorithm. Then, the numerical procedure is implemented in a computer code.

Some applications are presented. The axial and the bending response of the reinforced concrete and masonry beam are investigated. Moreover, several loading histories are considered. Some comparisons between analytical and numerical solutions are developed in order to assess the efficiency of the procedure.

The paper is organized as follows. Initially the proposed elastoplastic-damage model is described. Then, the crosssection beam equations are deduced. The numerical procedure and the arc-length technique are presented in detail. Finally, some numerical applications on reinforced concrete and masonry elements are reported.

\section{CONSTITUTIVE MODEL}

A thermodynamically consistent one-dimensional constitutive relation is addressed. The free energy is assumed to be:

$$
\begin{aligned}
\psi= & \eta\left[\frac{1}{2}\left(1-D^{+}\right) E\left(\varepsilon-\varepsilon_{p}\right)^{2}+g^{+}\left(\xi^{+}\right)\right] \\
& +(1-\eta)\left[\frac{1}{2}\left(1-D^{-}\right) E\left(\varepsilon-\varepsilon_{p}\right)^{2}+g^{-}\left(\xi^{-}\right)+k(\beta)\right]
\end{aligned}
$$

where $E$ is the Young modulus of the material, $D^{+}$and $D^{-}$are the damage parameters in tension and in compression, respectively, satisfying the classical inequalities $0 \leq D^{ \pm} \leq 1$, with $D^{ \pm}=0$ for the virgin material and $D^{ \pm}=1$ for the completely damaged material; $\varepsilon$ is the total strain, $\varepsilon_{p}$ is the plastic strain, so that $\varepsilon-\varepsilon_{p}=\varepsilon_{e}$ represents the elastic strain; $\xi^{+}$and $\xi^{-}$ are the internal parameters governing the damage softening in tension and in compression, respectively, $\beta$ is the internal parameter governing the plasticity hardening and $\eta$ is the stepwise function of the elastic strain $\varepsilon_{e}$, such that $\eta=1$ if $\varepsilon_{e} \geq 0$ and $\eta=0$ if $\varepsilon_{e}<0$. Note that in the following, the superscript ${ }^{+}$ 
corresponds to the case $\eta=1$, i.e. $\varepsilon_{e} \geq 0$, and the superscript ${ }^{-}$ corresponds to the case $\eta=0$, i.e., $\varepsilon_{e}<0$.

It is assumed that $D^{+} \geq D^{-}$that means that, on the base of experimental results, the damage in tension does not lead damage in compression, while the damage in compression induces a degradation of the material properties also in tension.

The functions $g^{ \pm}\left(\xi^{ \pm}\right)$and $k(\beta)$ are set as:

$$
\begin{aligned}
g^{ \pm}\left(\xi^{ \pm}\right) & =\frac{1}{2} E \frac{\left(\varepsilon_{c}^{ \pm}\right)^{2}}{\left(1+\alpha^{ \pm} \xi^{ \pm}-\xi^{ \pm}\right)\left(\alpha^{ \pm}-1\right)} \\
k(\beta) & =\frac{1}{2} K \beta^{2}
\end{aligned}
$$

where $\varepsilon_{c}^{ \pm}$represents the starting damage threshold strain, $\alpha^{ \pm}=$ $\varepsilon_{c}^{ \pm} / \varepsilon_{u}^{ \pm}$is the threshold ratio, with $\varepsilon_{u}^{ \pm}$the final damage threshold strain, and $K$ is the plastic hardening parameter. The threshold strains $\varepsilon_{c}^{ \pm}$and $\varepsilon_{u}^{ \pm}$and the plastic hardening quantity $K$ are material parameters.

The state laws are obtained deriving the free energy respect to the internal variables:

$$
\begin{aligned}
\sigma= & \frac{\partial \psi}{\partial \varepsilon}=\eta\left[\left(1-D^{+}\right) E\left(\varepsilon-\varepsilon_{p}\right)\right]+(1-\eta) \\
& \times\left[\left(1-D^{-}\right) E\left(\varepsilon-\varepsilon_{p}\right)\right] \\
Y^{ \pm}= & -\frac{\partial \psi}{\partial D^{ \pm}}=\frac{1}{2} E\left(\varepsilon-\varepsilon_{p}\right)^{2}=\frac{1}{2} E \varepsilon_{e}^{2}=Y \\
\zeta^{ \pm}=- & \frac{\partial \psi}{\partial \xi^{ \pm}}=\frac{1}{2} E \frac{\left(\varepsilon_{c}^{ \pm}\right)^{2}}{\left(1+\alpha^{ \pm} \xi^{ \pm}-\xi^{ \pm}\right)^{2}} \\
\tau=- & \frac{\partial \psi}{\partial \varepsilon_{p}}=\sigma \\
\vartheta= & -\frac{\partial \psi}{\partial \beta}=-(1-\eta) K \beta
\end{aligned}
$$

where $\sigma$ is the stress, $Y$ is the damage energy release rate, $\zeta^{ \pm}$ is the thermodynamical force associated to $\xi^{ \pm}, \tau$ is the thermodynamical force associated to the plastic strain and, indeed, it results $\tau=\sigma$, finally $\vartheta$ is the hardening plastic force.

The evolutionary equations of the internal damage and plastic state variables $D^{ \pm}, \xi^{ \pm}, \varepsilon_{p}, \beta$ are evaluated introducing two damage yield functions $f^{+}$and $f^{-}$and a plastic yield function $f^{p}$. In particular, the damage evolution is governed by the following equations:

$$
\begin{aligned}
f^{ \pm}\left(Y, \zeta^{ \pm}\right) & =Y-\zeta^{ \pm} \leq 0 \quad \dot{\gamma}^{ \pm} \geq 0 \quad f^{ \pm} \dot{\gamma}^{ \pm}=0 \\
\dot{D}^{ \pm} & =\frac{\partial f^{ \pm}}{\partial Y} \dot{\gamma}^{ \pm}=\dot{\gamma}^{ \pm} \\
\dot{\xi}^{ \pm} & =-\frac{\partial f^{ \pm}}{\partial \zeta^{ \pm}} \dot{\gamma}^{ \pm}=\dot{\gamma}^{ \pm}
\end{aligned}
$$

with $\dot{\gamma}^{ \pm}$the loading/unloading damage multiplier. From Eq. (5), it results that the parameter $\xi^{ \pm}$coincides with the damage internal state variable $D^{ \pm}$, in fact $\dot{D}^{ \pm}=\dot{\xi}^{ \pm}=\dot{\gamma}^{ \pm}$.
Taking into account the state law Eq. (4), the limit condition $(5)_{1}$ can be rewritten as:

$$
0=Y-\zeta^{ \pm}=\frac{1}{2} E \varepsilon_{e}^{2}-\frac{1}{2} E \frac{\varepsilon_{c}^{ \pm}}{\left(1+\alpha^{ \pm} D^{ \pm}-D^{ \pm}\right)^{2}}
$$

which, considering a monotonic damage evolution, leads to:

$$
\varepsilon_{e}=\frac{\varepsilon_{c}^{ \pm}}{1+\alpha^{ \pm} D^{ \pm}-D^{ \pm}}
$$

i.e.,

$$
D^{ \pm}=\frac{\varepsilon_{c}^{ \pm}-\varepsilon_{e}}{\left(\alpha^{ \pm}-1\right) \varepsilon_{e}}=\varepsilon_{u} \frac{\varepsilon_{c}^{ \pm}-\varepsilon_{e}}{\left(\varepsilon_{c}^{ \pm}-\varepsilon_{u}^{ \pm}\right) \varepsilon_{e}}
$$

Thus, it results $D^{ \pm}=0$ for $\varepsilon_{e}=\varepsilon_{c}^{ \pm}$and $D^{ \pm}=1$ for $\varepsilon_{e}=\varepsilon_{u}^{ \pm}$; moreover, substituting the deduced relation (8) into the expression of the stress given by the first equation of the state laws $(4)_{1}$, a stress-strain linear softening is obtained when no plastic evolution is considered.

The evolution of the damage parameter $\dot{D}^{ \pm}=\dot{\xi}^{ \pm}=\dot{\gamma}^{ \pm}$is computed from the consistency equations. In fact, it is:

$$
\begin{gathered}
0=\dot{f}^{ \pm}\left(Y, \zeta^{ \pm}\right)=E \varepsilon_{e} \dot{\varepsilon}_{e}+E \frac{\left(\varepsilon_{c}^{ \pm}\right)^{2}\left(\alpha^{ \pm}-1\right)}{\left(1+\alpha^{ \pm} \xi^{ \pm}-\xi^{ \pm}\right)^{3}} \dot{\xi}^{ \pm} \\
\text {with } f^{ \pm}=0 \text { and } \dot{\zeta}^{ \pm}>0
\end{gathered}
$$

Equation (9) is rewritten considering the definitions of the thermodynamical force $\zeta^{ \pm}$and of the damage energy release rate $Y$; recalling that $\xi^{ \pm}=D^{ \pm}$, it results:

$$
\begin{aligned}
& 0=\dot{\varepsilon}_{e}+\frac{\left(\alpha^{ \pm}-1\right) \varepsilon_{e}}{\left(1+\alpha^{ \pm} D^{ \pm}-D^{ \pm}\right)} \dot{D}^{ \pm} \quad \text { with } \quad f^{ \pm}=0 \\
& \text { and } \dot{D}^{ \pm}>0
\end{aligned}
$$

Substituting the damage parameter given from formula (8) into Eq. (10), the damage rate can be written as function of the elastic strain rate:

$$
\dot{D}^{ \pm}=\frac{\varepsilon_{c}^{ \pm}}{\left(1-\alpha^{ \pm}\right) \varepsilon_{e}^{2}} \dot{\varepsilon}_{e} \quad \text { with } \quad f^{ \pm}=0 \quad \text { and } \quad \dot{D}^{ \pm}>0
$$

The plasticity evolution is modeled introducing the following loading/unloading conditions:

$f^{p}(\sigma, \vartheta)=-\frac{\sigma}{1-D^{-}}+\vartheta-\sigma_{y} \leq 0 \quad \dot{\mu} \geq 0 \quad f^{p} \dot{\mu}=0$

with $\dot{\mu}$ the plastic multiplier and $\sigma_{y}>0$ the limit plastic stress in compression. It is fair to note that the plastic yield function can be active only in compression. The evolutions of the plastic strain and of the hardening variable are governed by associated 
laws:

$$
\dot{\varepsilon}_{p}=\frac{\partial f^{p}}{\partial \sigma} \dot{\mu}=-\frac{\dot{\mu}}{1-D^{-}} \quad \dot{\beta}=\frac{\partial f^{p}}{\partial \vartheta} \dot{\mu}=\dot{\mu}
$$

From Eq. (13) it results:

$$
\dot{\beta}=-\left(1-D^{-}\right) \dot{\varepsilon}_{p}
$$

In Eq. (12) the quantity $\sigma /(1-D)=\tilde{\sigma}$ is the effective stress in compression.

The consistency condition for the plastic process leads to:

$$
\begin{aligned}
0= & \dot{f}^{p}(\sigma, \vartheta)=\frac{\partial f^{p}}{\partial \sigma} \dot{\sigma}+\frac{\partial f^{p}}{\partial \vartheta} \dot{\vartheta}=E\left(\dot{\varepsilon}-\dot{\varepsilon}_{p}\right) \\
& +K\left(1-D^{-}\right) \dot{\varepsilon}_{p}
\end{aligned}
$$

The evolution of the plastic strain $\dot{\varepsilon}_{p}$ and, consequently of the hardening parameter $\dot{\beta}$, is obtained from Eq. (15):

$$
\dot{\varepsilon}_{p}=H \dot{\varepsilon} \quad \dot{\beta}=-\left(1-D^{-}\right) H \dot{\varepsilon}
$$

where

$$
H=\frac{E}{E+K\left(1-D^{-}\right)}
$$

Once the plastic strain evolution is determined in terms of the total strain rate, also the damage rate can be expressed in terms of the total strain rate as:

$$
\begin{aligned}
\dot{D}^{ \pm} & =\frac{\varepsilon_{c}^{ \pm}}{\left(1-\alpha^{ \pm}\right) \varepsilon_{e}^{2}}\left(\dot{\varepsilon}-\dot{\varepsilon}_{p}\right) \\
& =\frac{\varepsilon_{c}^{ \pm}}{\left(1-\alpha^{ \pm}\right) \varepsilon_{e}^{2}}(1-(1-\eta) H) \dot{\varepsilon}
\end{aligned}
$$

The tangent constitutive modulus $E_{T}^{ \pm}$is obtained by differentiating the stress-strain relationship:

$$
E_{T}^{ \pm}=E\left[\left(1-D^{ \pm}\right)-\frac{\varepsilon_{c}^{ \pm}}{\left(1-\alpha^{ \pm}\right) \varepsilon_{e}}\right](1-(1-\eta) H)
$$

Once the state laws and the damage and plastic evolution equations of the model have been defined, considering isothermal processes, the mechanical dissipation can be written in terms of the free energy as:

$$
\begin{aligned}
\mathrm{D}= & -\dot{\psi}+\sigma \dot{\varepsilon}=Y\left(\dot{D}^{+}+\dot{D}^{-}\right)+\zeta^{+} \dot{\xi}^{+} \\
& +\zeta^{-} \dot{\xi}^{-}+\sigma \dot{\varepsilon}_{p}+\vartheta \dot{\beta}
\end{aligned}
$$

It can be proved that, because of the definitions (4) and the formulas (5) and (13), the mechanical dissipation results always not negative, i.e., $\mathrm{D} \geq 0$, so that the Clausius-Duhem inequality is satisfied.

\section{CROSS-SECTION BEAM EQUATIONS}

The one-dimensional elastoplastic-damage constitutive law, developed in the previous section, is adopted to study the behavior of softening beams made of concrete or masonry. In particular, beam cross-sections, presenting a symmetry axis $y$, are considered. Note that the study can be extended to other geometries of the cross-section.

The possibility to account for the presence of elastic reinforcements is considered. Reinforcements are external, as the ones adopted to rehabilitate damaged concrete or masonry beams by using fiber-reinforced plastic materials.

The response of the damage-plastic cross-section beam is derived considering the elongation $e$ and the bending curvature $\chi$ deformations, such that the strain at a typical point of the beam is:

$$
\varepsilon=e+y \chi
$$

The resultants in the softening beam are:

$$
N^{B}(e, \chi)=\int_{A} \sigma(e, \chi) d A \quad M^{B}(e, \chi)=\int_{A} y \sigma(e, \chi) d A
$$

where $A$ is the cross-section area of the beam. Moreover, the resultants in the elastic reinforcements are:

$$
N^{R}=A^{R} e+B^{R} \chi \quad M^{R}=B^{R} e+D^{R} \chi
$$

where

$$
A^{R}=\sum_{i=1}^{n_{R}} E_{i}^{R} S_{i} \quad B^{R}=\sum_{i=1}^{n_{R}} E_{i}^{R} S_{i} h_{i} \quad D^{R}=\sum_{i=1}^{n_{R}} E_{i}^{R} S_{i} h_{i}^{2}
$$

with $n_{R}$ the number of reinforcements, $E_{i}^{R}, S_{i}$ and $h_{i}$ the Young modulus, the area and the abscissa of the $i$-th reinforcement, respectively.

The total resultant axial force and the bending moment in the reinforced beam are:

$$
N=N^{B}+N^{R} \quad M=M^{B}+M^{R}
$$

Finally, the behavior of the cross-section softening beam is governed by the equation:

$$
\begin{aligned}
R_{N}(e, \chi, \lambda) & =N(e, \chi)-\lambda N_{e x t} \\
& =N^{B}(e, \chi)+N^{R}(e, \chi)-\lambda N_{\text {ext }}=0 \\
R_{M}(e, \chi, \lambda) & =M(e, \chi)-\lambda M_{\text {ext }} \\
& =M^{B}(e, \chi)+M^{R}(e, \chi)-\lambda M_{\text {ext }}=0
\end{aligned}
$$

where $\lambda$ is the loading multiplier, introduced with the aim of developing an arc-length procedure. Equation (26) can be rewritten 
in vectorial form as:

$$
\mathbf{R}(\mathbf{u}, \lambda)=\mathbf{S}(\mathbf{u})-\lambda \omega=0
$$

where $\mathbf{u}$ is the beam elongation and curvature vector, $\mathbf{S}(\mathbf{u})$ is the internal stress resultant vector and $\omega$ is the external load vector; in particular, it is set:

$$
\mathbf{u}=\left[\begin{array}{l}
e \\
\chi
\end{array}\right] \quad \mathbf{S}(\mathbf{u})=\left[\begin{array}{l}
N(e, \chi) \\
M(e, \chi)
\end{array}\right] \quad \mathbf{\omega}=\left[\begin{array}{l}
N_{\text {ext }} \\
M_{\text {ext }}
\end{array}\right]
$$

\section{COMPUTATIONAL PROCEDURE}

The nonlinear equilibrium Eq. (27) is solved developing a numerical procedure. In particular, to evaluate the kinematic vector $\mathbf{u}$ and the loading multiplier $\lambda$, it is required:

- the time discretization of the equilibrium Eq. (27),

- the definition of the arc-length constrain equation and the setting of the control parameters,

- the time integration of the constitutive equations.

\subsection{Equilibrium Equations}

The following notation is adopted: the subscript ${ }_{n}$ indicates a quantity evaluated at time $t_{n}$ while no subscript indicates a quantity evaluated at time $t_{n+1}$. Moreover, $\Delta$ indicates the variable increment at the time step $\Delta t$. It is assumed that the solution $\mathbf{u}_{n}$, i.e. the beam elongation and curvature vector at the time $t_{n}$ corresponding to the load parameter $\lambda_{n}$, is known. A new equilibrium configuration at a subsequent time $t_{n+1}$, is given by:

$$
\mathbf{u}=\mathbf{u}_{n}+\Delta \mathbf{u} \quad \lambda=\lambda_{n}+\Delta \lambda
$$

such that Eq. (27) can be written as:

$$
\mathbf{R}(\mathbf{u}, \lambda)=\mathbf{S}\left(\mathbf{u}_{n}+\Delta \mathbf{u}\right)-\left(\lambda_{n}+\Delta \lambda\right) \boldsymbol{\omega}=\mathbf{0}
$$

The Newton-Raphson algorithm is adopted to solve the finite step equilibrium problem (30), which is written in a linearized form:

$$
\mathbf{R}\left(\mathbf{u}^{k+1}, \lambda^{k+1}\right)=\mathbf{R}\left(\mathbf{u}^{k}, \lambda^{k}\right)+\mathbf{K}_{t} \delta \mathbf{u}-\boldsymbol{\omega} \delta \lambda=\mathbf{0}
$$

where $\delta \mathbf{u}=\mathbf{u}^{k+1}-\mathbf{u}^{k}, \delta \lambda=\lambda^{k+1}-\lambda^{k}$, the superscripts ${ }^{k}$ and ${ }^{k+1}$ indicate the iteration indices and $\mathbf{K}_{t}$ is the tangent matrix, defined as:

$$
\mathbf{K}_{t}=\left.\frac{\partial \mathbf{R}}{\partial \mathbf{u}}\right|_{\mathbf{u}=\mathbf{u}^{k}}=\left[\begin{array}{cc}
\frac{\partial R_{N}}{\partial e} & \frac{\partial R_{N}}{\partial \chi} \\
\frac{\partial R_{M}}{\partial e} & \frac{\partial R_{M}}{\partial \chi}
\end{array}\right]=\left[\begin{array}{cc}
A^{B}+A^{R} & B^{B}+B^{R} \\
B^{B}+B^{R} & D^{B}+D^{R}
\end{array}\right]
$$

with

$$
\begin{aligned}
A^{B} & =\int_{A} \frac{\partial \sigma}{\partial e} d A \quad B^{B}=\int_{A} \frac{\partial \sigma}{\partial \chi} d A=\int_{A} y \frac{\partial \sigma}{\partial e} d A \\
D^{B} & =\int_{A} y \frac{\partial \sigma}{\partial \chi} d A
\end{aligned}
$$

The tangent moduli $\partial \sigma / \partial e$ and $\partial \sigma / \partial \chi$ are computed as:

$$
\frac{\partial \sigma}{\partial e}=E_{T}^{ \pm} \quad \frac{\partial \sigma}{\partial \chi}=y E_{T}^{ \pm}
$$

Substituting the tangent moduli into Eq. (33), the explicit form for the quantities $A^{B}, B^{B}$ and $D^{B}$ is obtained:

$$
A^{B}=\int_{A} E_{T}^{ \pm} d A \quad B^{B}=\int_{A} y E_{T}^{ \pm} d A \quad D^{B}=\int_{A} y^{2} E_{T}^{ \pm} d A
$$

Once $A^{B}, B^{B}$ and $D^{B}$ are determined, the tangent matrix $\mathbf{K}_{t}$, defined by formula (32), can be explicitly evaluated.

Note that, the integration over the cross-section to determine the residuals $\mathbf{R}$ and the quantities $A^{B}, B^{B}$ and $D^{B}$ can be performed by discretizing the cross-section in stripes and applying the Gauss integration formula within each stripe, which can be considered as an extension of the classical fiber model. The time integration of the local constitutive equations is required to define the damage and plastic evolutions in each Gauss point. This aspect is described in detail in Section 4.3.

\subsection{Arc-length Procedure}

The softening behavior of the material constituting the beam can induce an overall response characterized by steep softening and snap-back branches. Hence, it appears convenient to adopt an arc-length method able to catch the overall beam response. In particular, the cylindrical as well as the linearized arc-length methods [26] with local control are developed for the particular problem under consideration.

Special attention is addressed to the choice of the control parameters which represents a key point of the arc-length method [27]. The two strains $\hat{\varepsilon}^{+}$and $\hat{\varepsilon}^{-}$, evaluated at $y=y_{u}^{+}$and $y=$ $y_{u}^{-}$, are assumed as control parameters. The coordinates $y_{u}^{+}$and $y_{u}^{-}$define the position of the axes in the cross-section where the elastic strains computed at the time $t_{n}$ are equal to the tensile and compressive final damage threshold strains, i.e., $\varepsilon_{e, n}\left(y_{u}^{+}\right)=\varepsilon_{u}^{+}$ and $\varepsilon_{e, n}\left(y_{u}^{-}\right)=\varepsilon_{u}^{-}$. They are determined as:

$$
\begin{gathered}
y_{u}^{+}: e_{n}+y_{u}^{+} \chi_{n}-\varepsilon_{p, n}\left(y_{u}^{+}\right)=\varepsilon_{u}^{+} \\
\Rightarrow y_{u}^{+}=\frac{\varepsilon_{u}^{+}-e_{n}+\varepsilon_{p, n}\left(y_{u}^{+}\right)}{\chi_{n}} \\
y_{u}^{-}: e_{n}+y_{u}^{-} \chi_{n}-\varepsilon_{p, n}\left(y_{u}^{-}\right)=\varepsilon_{u}^{-} \\
\Rightarrow y_{u}^{-}=\frac{\varepsilon_{u}^{-}-e_{n}+\varepsilon_{p, n}\left(y_{u}^{-}\right)}{\chi_{n}}
\end{gathered}
$$


It can be noted that, applying formula (36), the coordinates $y_{u}^{+}$ and $y_{u}^{-}$could be not internal to the cross-section, for any possible loading conditions. In that cases, it is assumed $y_{u}^{+}= \pm h / 2$ and $y_{u}^{-}=\mp h / 2$, where $h$ is the height of the cross-section along the $y$ axis and the sign + or - is selected in dependence on the sign of the bending moment. Finally, as announced above, the arc-length procedure is controlled by the increment of the strains $\hat{\varepsilon}^{+}=\varepsilon\left(y_{u}^{+}\right)$and $\hat{\varepsilon}^{-}=\varepsilon\left(y_{u}^{-}\right)$.

According to the cylindrical version of the arc-length method, the constraint equation in the finite step is:

$$
\ell^{2}=\left[\Delta \hat{\varepsilon}^{+}(\delta \mathbf{u}, \delta \lambda)\right]^{2}
$$

where $\ell$ is the prescribed incremental solution length.

The solution of Eqs. (31) and (37) allows to compute the iterative kinematic increment $\delta \mathbf{u}$ and the multiplier load increment $\delta \lambda$. In particular, solving Eq. (31) with respect to $\delta \mathbf{u}$ and substituting the deduced expression into Eq. (37), the classical algebraic quadratic equation of the cylindrical arc-length method is obtained. The iterative load factor is chosen as the solution of the quadratic equation that yields the minimum angle between $\mathbf{u}^{k}$ and $\mathbf{u}^{k+1}$.

\subsection{Time Integration of the Constitutive Equations}

The time integration of Eqs. (16) and (18) in the interval $\left[t_{n}, t_{n+1}\right]$ is performed adopting a backward-Euler scheme [28]. The evaluation of the plastic and damage increments in the finite time step is performed setting:

$$
\begin{gathered}
\Delta \varepsilon_{p}=\int_{t_{n}}^{t_{n+1}} \dot{\varepsilon}_{p} d t=\varepsilon_{p}-\varepsilon_{p, n} \\
\Delta D^{ \pm}=\int_{t_{n}}^{t_{n+1}} \dot{D}^{ \pm} d t=D^{ \pm}-D_{n}^{ \pm}
\end{gathered}
$$

The discretized form of the evolutionary equations of the plastic strain (16) and of the damage (18) are:

$$
\begin{aligned}
\varepsilon_{p} & =\varepsilon_{p, n}+H \Delta \varepsilon \\
D^{ \pm} & =D_{n}^{ \pm}+\frac{\varepsilon_{c}^{ \pm}}{\left(1-\alpha^{ \pm}\right)\left(\varepsilon-\varepsilon_{p}\right)}(1-(1-\eta) H) \Delta \varepsilon
\end{aligned}
$$

where $H$ is defined by formula (17). The solution of the coupled Eqs. (39) is performed by means of a return-mapping algorithm, i.e. a predictor-corrector procedure [28]. The trial elastic predictor phase is evaluated keeping frozen the plastic strain and the damage obtained at time $t_{n}$ :

$$
\begin{aligned}
\varepsilon_{p}^{t r} & =\varepsilon_{p, n} \\
\beta^{t r} & =\beta_{n} \\
D^{ \pm, t r} & =D_{n}^{ \pm} \\
\sigma^{t r} & =\left(1-D^{ \pm, t r}\right) E\left(\varepsilon-\varepsilon_{p}^{t r}\right)
\end{aligned}
$$

$$
\begin{aligned}
\vartheta^{t r} & =-K \beta^{t r} \\
f^{p, t r} & =-\frac{\sigma^{t r}}{\left(1-D^{-, t r}\right)}+\vartheta-\sigma_{y}=E\left(\varepsilon-\varepsilon_{p}^{t r}\right)-K \beta^{t r}-\sigma_{y} \\
f^{ \pm, t r} & =\frac{E}{2}\left[\left(\varepsilon-\varepsilon_{p}^{t r}\right)^{2}-\frac{\left(\varepsilon_{c}^{ \pm}\right)^{2}}{\left(1+\alpha^{ \pm} D^{ \pm, t r}-D^{ \pm, t r}\right)^{2}}\right]
\end{aligned}
$$

Depending on the values of $f^{p, t r}$ and $f^{ \pm, t r}$, four different cases can occur.

Case $1 f^{p, t r}<0 \quad f^{ \pm, t r}<0$

The damage and yield functions are satisfied so there is neither plastic nor damage evolution. The elastic trial state is the solution of the damage plastic problem at this step.

Case $2 f^{p, t r}<0 \quad f^{ \pm, t r} \geq 0$

The damage limit function is not satisfied. In this case, only damage evolution arises; in traction, there is never plastic evolution; while in compression, it can be noted that the plastic yield function does not depend on the value of $D^{ \pm}$; thus, an increment of the variable $D^{ \pm}$, does not change the value of the plastic yield function, i.e., $f^{p}=f^{p, t r}<0$, so no plastic evolution occurs. Finally, the solution of Case 2 is computed solving Eq. (39) 2 considering $\varepsilon_{p}=\varepsilon_{p, n}, D^{ \pm} \geq D_{n}^{ \pm}$and $0 \leq D^{ \pm} \leq 1$. It results:

$$
\begin{aligned}
\varepsilon_{p} & =\varepsilon_{p, n} \\
\beta & =\beta_{n} \\
D^{ \pm} & =D_{n}^{ \pm}+\frac{\varepsilon_{c}^{ \pm}}{\left(1-\alpha^{ \pm}\right)\left(\varepsilon-\varepsilon_{p}\right)}(1-(1-\eta) H) \Delta \varepsilon
\end{aligned}
$$

Case $3 f^{p, t r} \geq 0 \quad f^{ \pm, t r}<0$

This case can occur only in compression. The plastic evolution is evaluated solving Eq. (39) $)_{1}$ with $D^{ \pm}=D_{n}^{ \pm}$. Once $\varepsilon_{p}$ is computed, the trial damage limit function $f^{-, t r}$ is evaluated. If $f^{-, t r}<0$, then, only plastic evolution occurs and the solution is:

$$
\begin{aligned}
D^{ \pm} & =D_{n}^{ \pm} \\
\varepsilon_{p} & =\varepsilon_{p, n}+H \Delta \varepsilon \\
\beta & =\beta_{n}-\left(1-D^{-}\right) H \Delta \varepsilon
\end{aligned}
$$

On the contrary, if $f^{-, t r} \geq 0$, also a damage evolution occurs and the evaluation of the plastic and damage increments is performed solving Case 4.

Case $4 f^{p, t r} \geq 0 \quad f^{-, t r} \geq 0$

Also this case can occur only in compression. The plastic strain and damage evolutions are evaluated solving the coupled nonlinear evolutive Eq. (39) under the constrains $\varepsilon_{p} \leq \varepsilon_{p, n}, D^{ \pm} \geq D_{n}^{ \pm}$ 
and $0 \leq D^{ \pm} \leq 1$. To this end, the Newton-Raphson algorithm is adopted. Equations (39) are rewritten in residual form:

$$
\begin{aligned}
r_{p} & =\varepsilon_{p}-\varepsilon_{p, n}-H \Delta \varepsilon \\
r_{D} & =D^{-}-D_{n}^{-}-\frac{\varepsilon_{c}^{ \pm}}{\left(1-\alpha^{ \pm}\right)\left(\varepsilon-\varepsilon_{p}\right)}(1-H) \Delta \varepsilon
\end{aligned}
$$

whose linearization gives:

$$
\left\{\begin{array}{l}
r_{p}\left(\varepsilon_{p}^{j+1}, D^{-, j+1}\right) \\
r_{D}\left(\varepsilon_{p}^{j+1}, D^{-, j+1}\right)
\end{array}\right\}=\left\{\begin{array}{l}
r_{D}\left(\varepsilon_{p}^{j}, D^{-, j}\right) \\
r_{D}\left(\varepsilon_{p}^{j}, D^{-, j}\right)
\end{array}\right\}+\mathbf{Z}_{t}^{j}\left\{\begin{array}{l}
\delta \varepsilon_{p} \\
\delta D^{-}
\end{array}\right\}=0
$$

where $\delta \varepsilon_{p}=\varepsilon_{p}^{j+1}-\varepsilon_{p}^{j}, \delta D^{-}=D^{-, j+1}-D^{-, j}$, the superscripts ${ }^{j}$ and ${ }^{j+1}$ indicate the iteration indices and the tangent matrix $\mathbf{Z}_{t}^{j}$ is:

$$
\mathbf{Z}_{t}^{j}=\left.\left[\begin{array}{ll}
\frac{\partial r_{p}}{\partial \varepsilon_{p}} & \frac{\partial r_{p}}{\partial D^{-}} \\
\frac{\partial r_{D}}{\partial \varepsilon_{p}} & \frac{\partial r_{D}}{\partial D^{-}}
\end{array}\right]\right|_{j}
$$

with

$$
\begin{aligned}
\left.\frac{\partial r_{p}}{\partial \varepsilon_{p}}\right|_{j} & =1 \\
\left.\frac{\partial r_{p}}{\partial D^{-}}\right|_{j} & =\frac{E K \Delta \varepsilon}{\left(E+K\left(1-D^{-, j}\right)\right)^{2}} \\
\left.\frac{\partial r_{D}}{\partial \varepsilon_{p}}\right|_{j} & =\frac{K\left(1-D^{-, j}\right) \varepsilon_{c}^{-} \Delta \varepsilon}{\left.\left(1-\alpha^{-}\right)\left(\varepsilon-\varepsilon_{p}^{j}\right)^{3}\left(E+K\left(1-D^{-, j}\right)\right)\right)^{2}} \\
\left.\frac{\partial r_{D}}{\partial D^{-}}\right|_{j} & =1+\frac{E K \varepsilon_{c}^{-} \Delta \varepsilon}{\left(1-\alpha^{-}\right)\left(\varepsilon-\varepsilon_{p}^{j}\right)^{2}\left(E+K\left(1-D^{-, j}\right)\right)^{2}}
\end{aligned}
$$

The damage $\delta D^{-}$and plastic $\delta \varepsilon_{p}$ increments are evaluated solving the system of Eq. (44). Then, the iterative procedure goes on until a convergence test is satisfied, i.e. when the values of the norm of the residual vector is less than a prefixed tolerance. When $D^{-}>D^{+}$it is set $D^{+}=D^{-}$.

\section{NUMERICAL APPLICATIONS}

Applications are developed to investigate the behavior of reinforced and unreinforced concrete and masonry rectangular cross-sections.

Initially, a masonry element reinforced by FRP sheets is analyzed. The axial and bending behavior is studied. Moreover, comparisons between the numerical and the analytical solutions are made in order to assess the efficiency of the procedure.

Then, the bending response of a concrete element, reinforced by FRP sheets is studied. Analyses are developed considering different constitutive laws for the concrete material, based on the prescriptions of the Eurocode 2 [29].

A regularized technique based on the fracture energy is adopted to derive the softening parameters for both the masonry and concrete numerical applications, considering the fracture energy equal to the damage dissipation in a volume of beam defined by the distance between two fractures.

\subsection{Reinforced Masonry Element}

The analyses are developed to study the mechanical response of a reinforced masonry element, characterized by the following material and geometrical data:

$$
\begin{aligned}
& E=5000 \mathrm{MPa} \quad \sigma_{y}=3 \mathrm{MPa} \quad K=500 \mathrm{MPa} \\
& \varepsilon_{c}^{+}=0.0001 \quad \varepsilon_{c}^{-}=-0.00067 \\
& \varepsilon_{u}^{+}=0.0004 \quad \varepsilon_{u}^{-}=-0.00085 \\
& E^{R}=200000 \mathrm{MPa} \\
& b=130 \mathrm{~mm} \quad h=250 \mathrm{~mm} \\
& n^{R}=2 \quad S_{1}=10 \mathrm{~mm}^{2} \quad S_{2}=10 \mathrm{~mm}^{2} \\
& h_{1}=125 \mathrm{~mm} \quad h_{2}=-125 \mathrm{~mm}
\end{aligned}
$$

where $b$ is the width of the cross-section.

Note that the material and geometrical data correspond to a possible masonry obtained using blocks with a rectangular cross-section of $130 \times 250 \mathrm{~mm}^{2}$, reinforced by low modulus carbon-epoxy composite material at the top and at the bottom of the cross-section.

Initially, the problem concerning the beam subjected to a tensile axial force $N_{e x t}$ and to a bending moment $M_{e x t}$, characterized by a prescribed eccentricity $d=M_{\text {ext }} / N_{\text {ext }}=25 \mathrm{~mm}$, is investigated. For the considered problem, the whole crosssection is in tension, thus only damage and no plasticity can occur; as a consequence, the analytical solution is determined by formulas reported in the appendix.

In Figures 1 and 2, the comparison between the analytical and the numerical solutions is shown. In particular, in Figure 1 the axial force versus the elongation is reported; while in Figure 2 the bending moment is plotted versus the curvature. It can be emphasized that the numerical procedure is able to compute a very satisfactory solution in terms of axial deformation as well as of curvature. In fact, the numerical solution is able to predict also sharp snap-back branches. These results point out that the choice of the control parameters in the arc-length method is appropriate. In Figures 1 and 2, the distributions of tensile stress along the masonry cross-section during the whole loading history are also schematically reported. It can be noted that the mechanical response of the reinforced section both in terms of axial force-elongation, reported in Figure 1, and bending momentcurvature, reported in Figure 2, is characterized by five different branches:

1. a linear elastic behavior when the whole section still behaves elastically, 


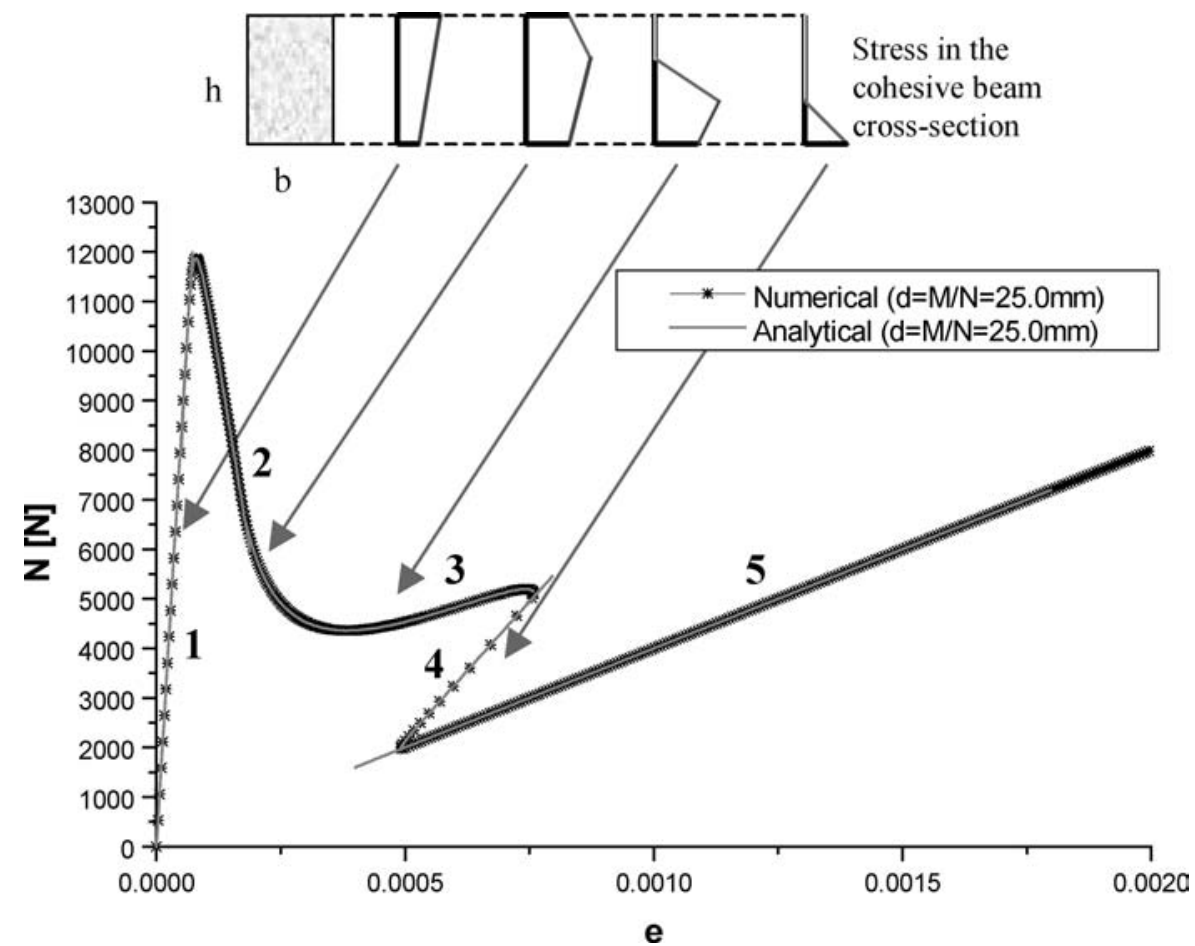

FIG. 1. Tensile axial force versus elongation: comparison between analytical and numerical solutions for $d=25 \mathrm{~mm}$.

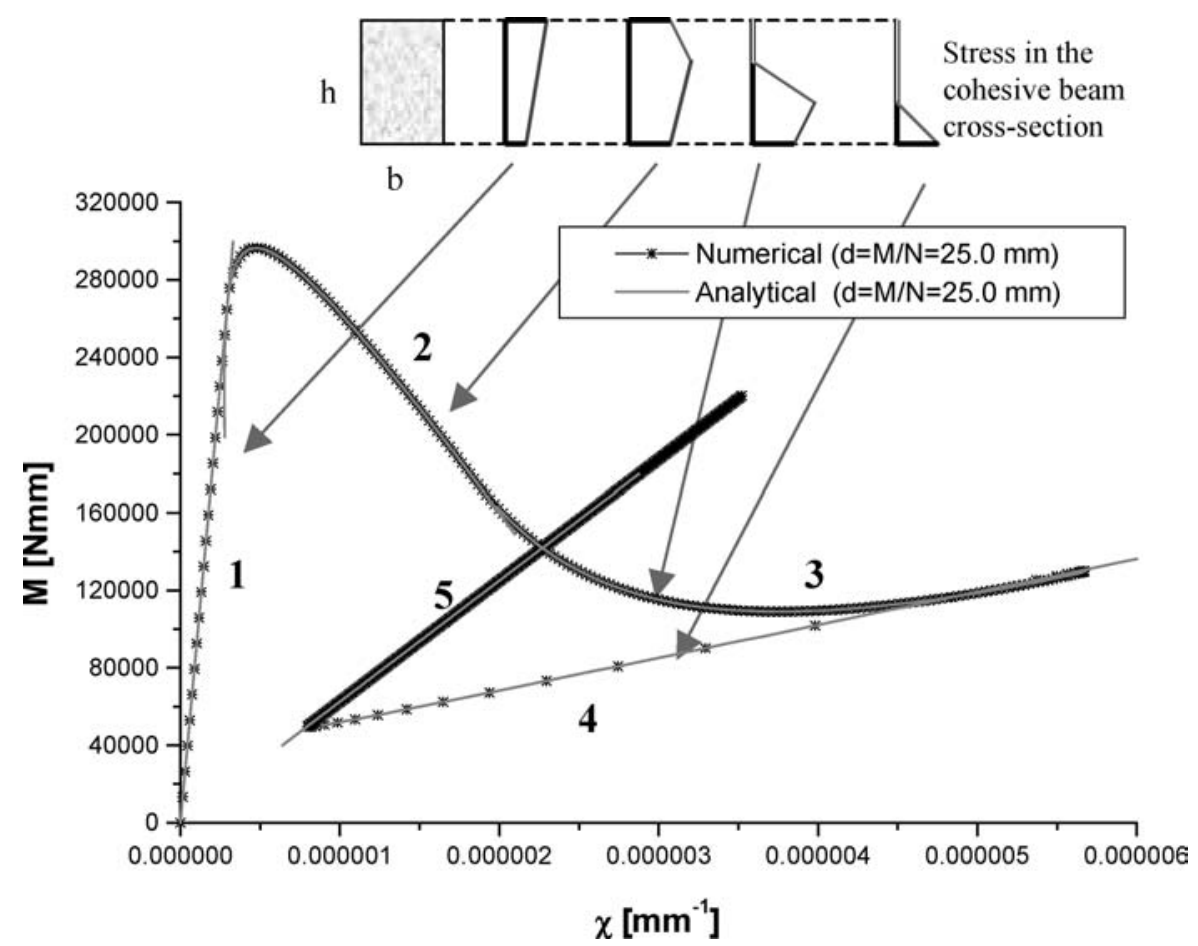

FIG. 2. Bending moment versus curvature: comparisons between analytical and numerical solutions for $d=25 \mathrm{~mm}$. 


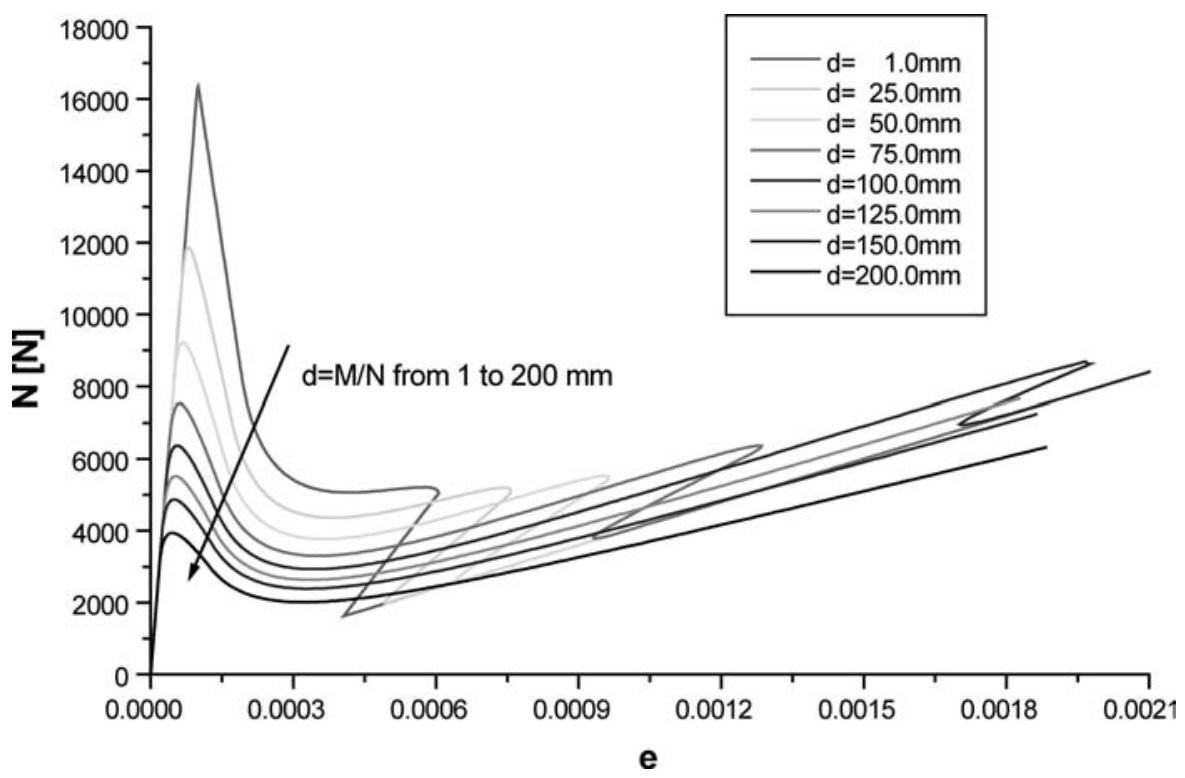

FIG. 3. Tensile axial force versus elongation for different values of the eccentricity $d$.

2. a softening branch after the peak that corresponds to the damage evolution in the upper part of the masonry section,

3. a hardening branch when the upper part of the section is completely damaged, the middle part is partially damaged and the lower one still behaves elastically,

4. a severe snap-back when the greater part of the masonry section is completely damaged and a smaller part is partially damaged,

5. a linear elastic branch that occurs when the whole masonry section is completely damaged and only the reinforcements are active.
Then, the beam, subjected to axial force and bending moment characterized by different values of the eccentricity $d$, is considered. In Figures 3 and 4, the tensile axial force versus the elongation and the bending moment versus the curvature are plotted, respectively. It can be noted that the response both in terms of axial force-elongation and bending moment-curvature is characterized by softening branches and severe snap-backs that correspond to the damage evolution in the masonry section. The mechanical response for any value of the eccentricity $d$ tends to the linear elastic behavior of the reinforcements when the cohesive material is completely damaged. As it can be seen

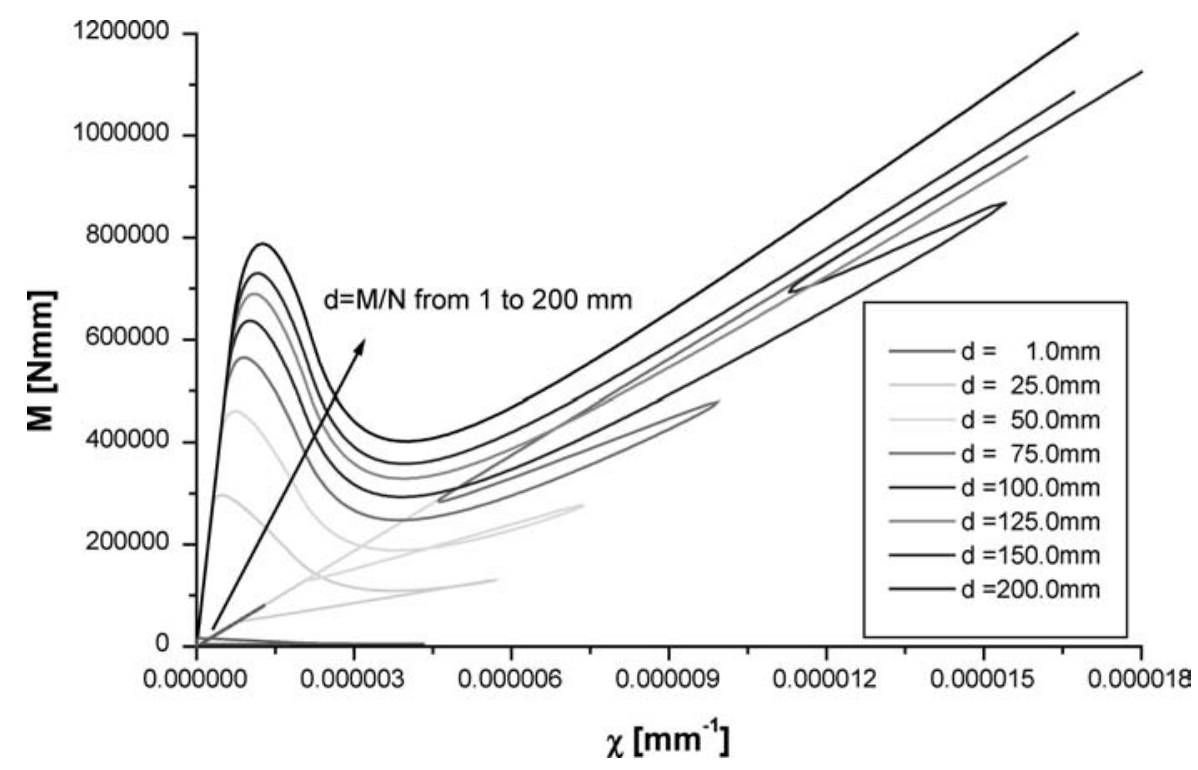

FIG. 4. Bending moment versus curvature for different values of the eccentricity $d$. 


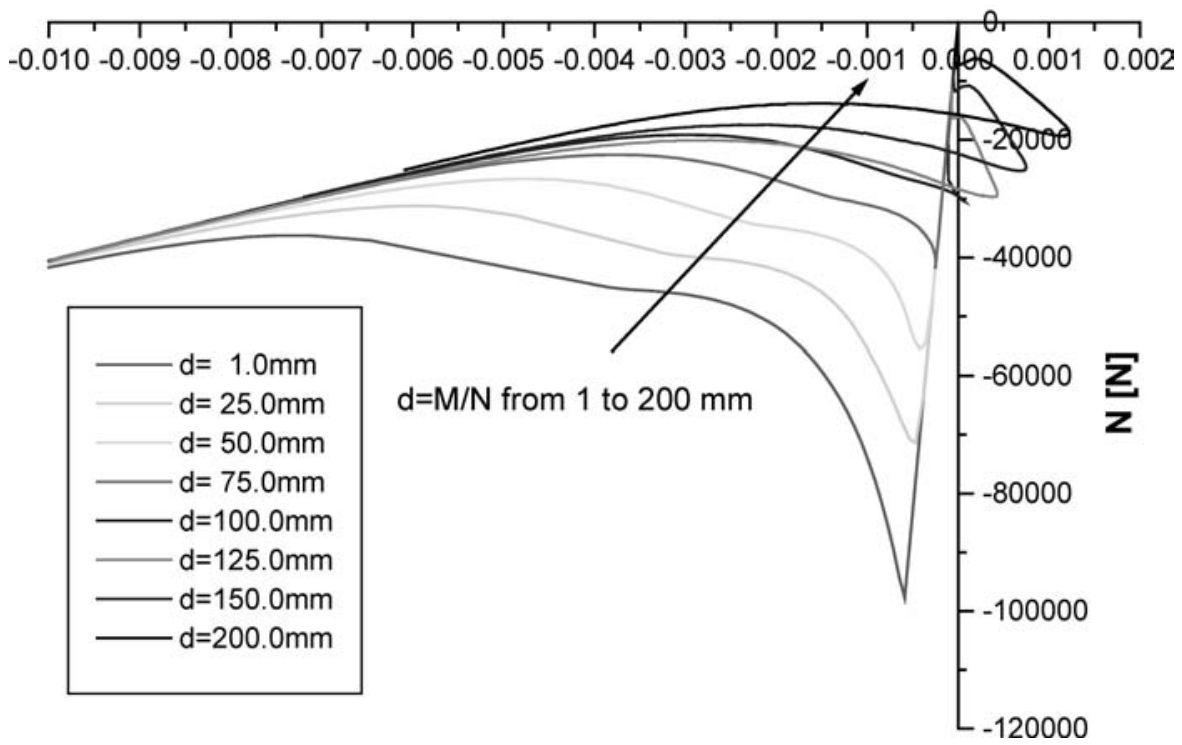

FIG. 5. Compressive axial force versus elongation for different values of the eccentricity $d$.

in Figure 3 and Figure 4, the mechanical response is characterized by severe snap-backs for small values of $d$, i.e., for high values of the maximum tensile axial force and small values of the maximum bending moment. The softening behavior is more regular without snap-backs for high values of $d$, i.e., for reduced values of the maximum tensile axial force and high values of the maximum bending moment, since in these cases the damage evolution occurs more gradually.

Moreover, in Figures 5 and 6, the compressive axial force versus the axial strain and the bending moment versus the curvature are plotted, respectively. In these cases, the response of the reinforced beam appears more complex as it is significantly influenced not only by the damage evolution but also by the plastic flow even for reduced values of the eccentricity $d$. The response of the beam for any loading history is characterized by steep softening branches and by severe snapbacks. As it can be seen in Figure 5, for high values of $d$ in the first part of the loading history a positive elongation occurs although the section is subjected to compressive axial force.

Finally, it can be pointed out that, also in these cases, the mechanical response tends to the linear elastic behavior of the

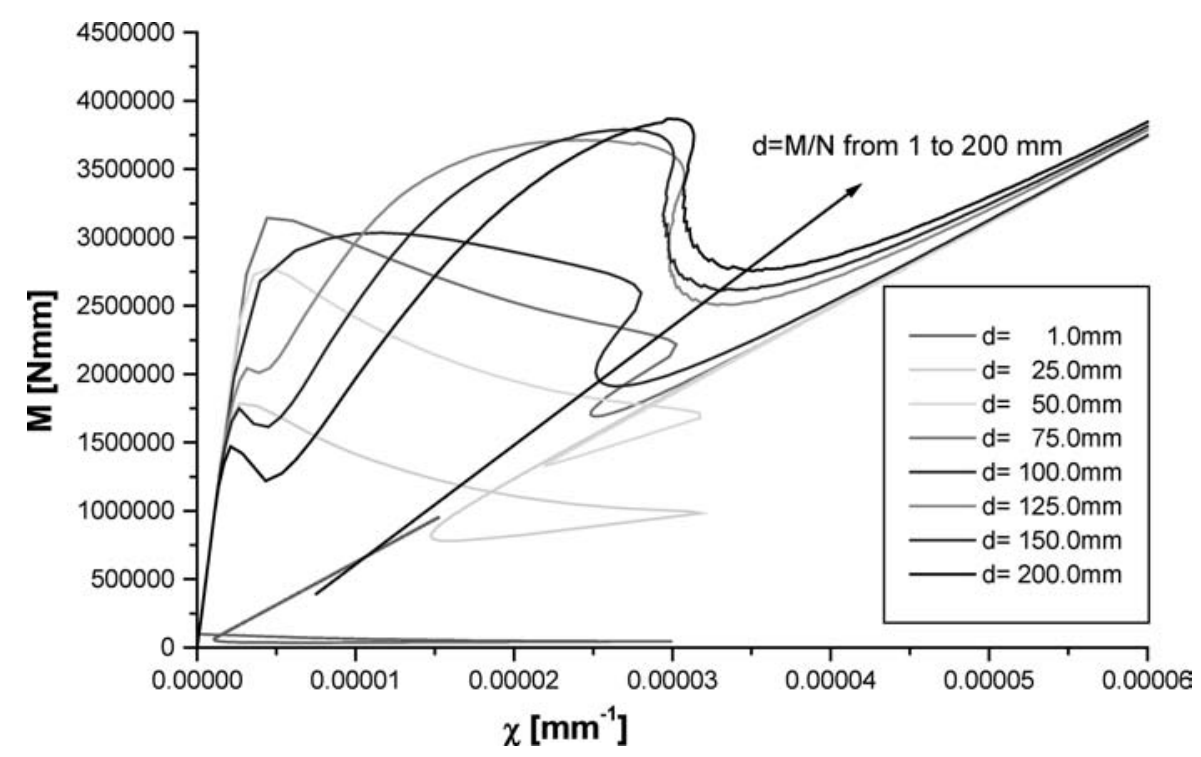

FIG. 6. Bending moment versus curvature for different values of the eccentricity $d$. 


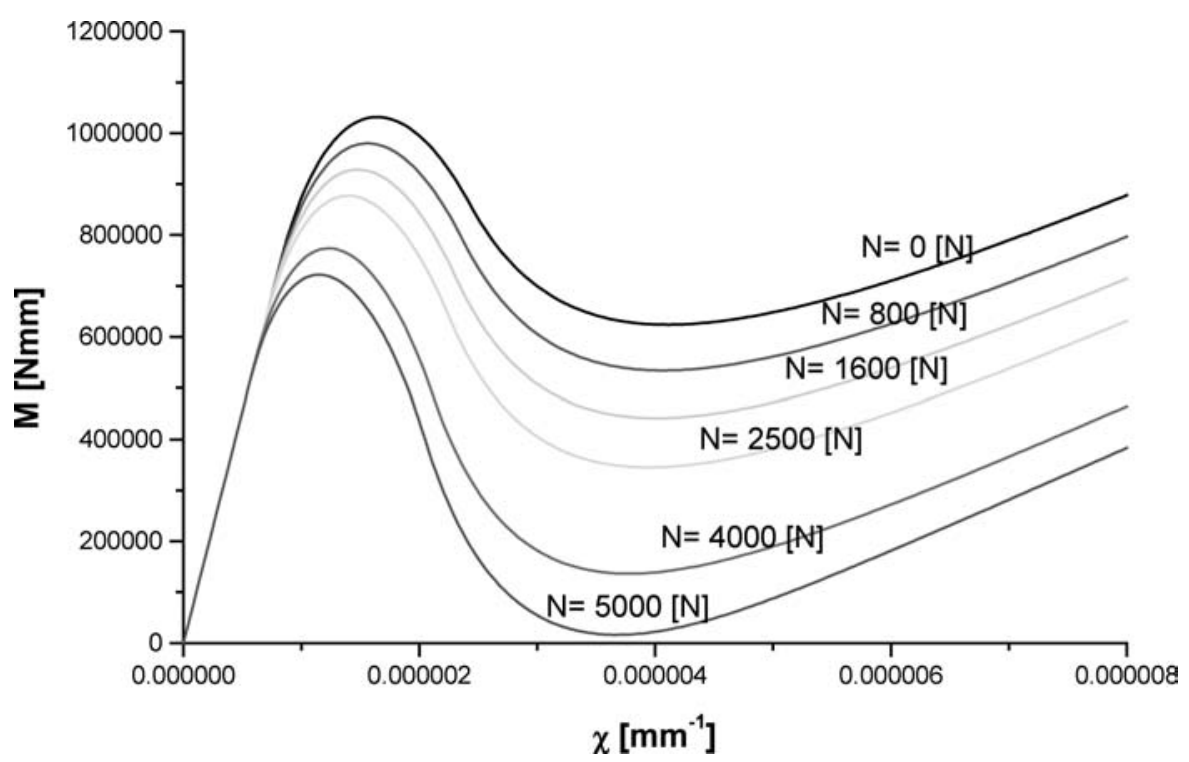

FIG. 7. Bending moment versus curvature for different values of the tensile axial force.

reinforcements for any value of $d$, when the masonry is completely damaged.

In Figures 7 and 8, the bending moment is plotted versus the curvature for different values of the tensile and compressive axial force, respectively. The considered loading histories consist in two steps: initially, the axial force is applied, such that $N_{\text {ext }}$ does not induce damage and plastic deformations in any part of the cross-section; then the bending moment is applied taking constant the axial force. From Figure 8, it is clear that the initial compression improves the structure response and bearing capabilities.

\subsection{FRP Reinforced Concrete Element}

A concrete element reinforced by FRP material is analyzed. In particular, it is studied a rectangular cross-section characterized by the following geometrical data:

$$
\begin{array}{rll}
b=300 \mathrm{~mm} & h=600 \mathrm{~mm} & \\
n^{R}=2 & S_{1}=125 \mathrm{~mm}^{2} & S_{2}=62 \mathrm{~mm}^{2} \\
& h_{1}=300 \mathrm{~mm} & h_{2}=-300 \mathrm{~mm}
\end{array}
$$

The amount of FRP considered in the cross-section of the concrete beam corresponds to 3 layers with a width of $250 \mathrm{~mm}$

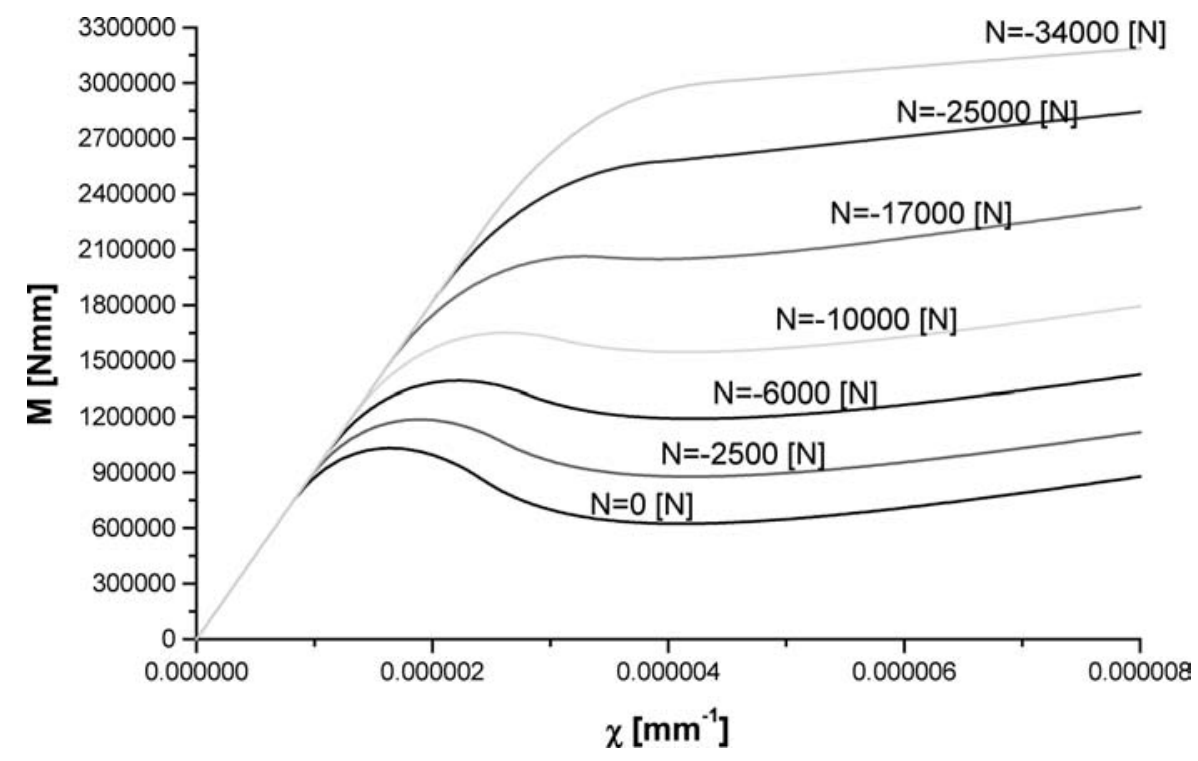

FIG. 8. Bending moment versus curvature for different values of the compressive axial force. 


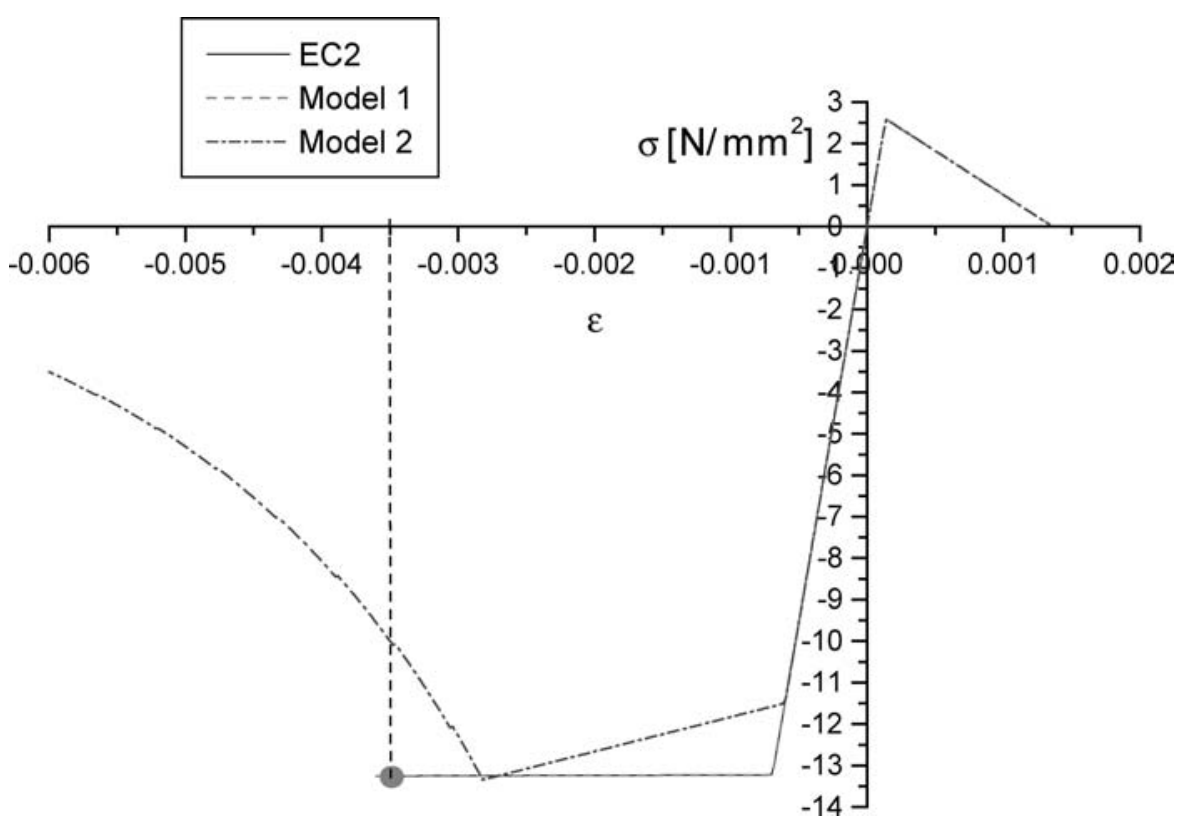

FIG. 9. Uniaxial stress-strain response for the EC2 model and for the proposed Model 1 and Model 2.

on the bottom and to 2 layers with a width of $180 \mathrm{~mm}$ on the top. A high modulus graphite FRP reinforcement, characterized by a Young modulus $E^{R}=760000 \mathrm{MPa}$, is considered for the analysis of concrete section. The FRP is modeled as a linear elastic material.

Three different concrete behaviors, schematically represented in Figure 9 are considered.

EC2 model considers a concrete response strictly following the Eurocode 2 [29] prescriptions. Hence, an elastoplastic behavior, with no hardening, is adopted in compression until the uniaxial deformation $\varepsilon$ reaches the limit value $\varepsilon_{f}=-0.0035$; moreover no strength in tension is assumed. The stress-strain relationship for EC2 model is schematically reported in Figure 9. The EC2 concrete response is recovered within the proposed elastoplastic-damage model setting the parameters as:

- compression

$$
\begin{array}{lr}
E=19050 \mathrm{MPa} \quad \sigma_{y}=13.226 \mathrm{MPa} & K=0 \mathrm{MPa} \\
\varepsilon_{c}^{-} \rightarrow \infty \quad \varepsilon_{u}^{-} \rightarrow \infty &
\end{array}
$$

- tension

$$
\begin{array}{ll}
E=19050 \mathrm{MPa} & \\
\varepsilon_{c}^{+} \rightarrow 0 & \varepsilon_{u}^{+} \rightarrow 0
\end{array}
$$

where the symbols $\rightarrow 0$ and $\rightarrow \infty$ indicate the assumption of very small and very high numerical values, respectively.

Note that the Young modulus $E$ and the yield stress $\sigma_{y}$ are determined following the prescriptions of the Eurocode 2.
Model 1 considers the same concrete response of EC2 model in compression, setting the model parameters as in (49); a finite strength, evaluated according to the Eurocode 2 [29] and a linear softening response are introduced in tension. In Figure 9 the stress-strain behavior, considered for Model 1, is reported. Model 1 concrete tensile response is obtained within the proposed model setting the parameters as:

$$
\begin{aligned}
E & =19050 \mathrm{MPa} \\
\varepsilon_{c}^{+} & =0.000136 \quad \varepsilon_{u}^{+}=0.00136
\end{aligned}
$$

Model 2 considers the damage response of Model 1 in tension, setting the model parameters as in (51); an elastoplastic-damage response is assumed in compression in order to better approximate the experimental behavior of concrete, as illustrated in Figure 9. Model 2 compressive response is recovered within the proposed model, setting the parameters as:

$$
\begin{aligned}
& E=19050 \mathrm{MPa} \quad \sigma_{y}=13.226 \mathrm{MPa} \quad K=870 \mathrm{MPa} \\
& \varepsilon_{c}^{-}=-0.00072 \quad \varepsilon_{u}^{-}=-0.00082
\end{aligned}
$$

The bending behavior of the reinforced concrete section is analyzed.

In Figure 10, the bending moment versus the curvature is plotted for the three different proposed concrete responses. It can be noted that there are significative differences, in the first part of the analyses, between the response obtained in EC2 model and in Model 1 and 2. In fact, the latter two stress-strain relationships are characterized by finite tensile strength, while a no tension material is assumed in EC2 model. In the second part of the analyses, when the material considered in Model 1 and 2 is 


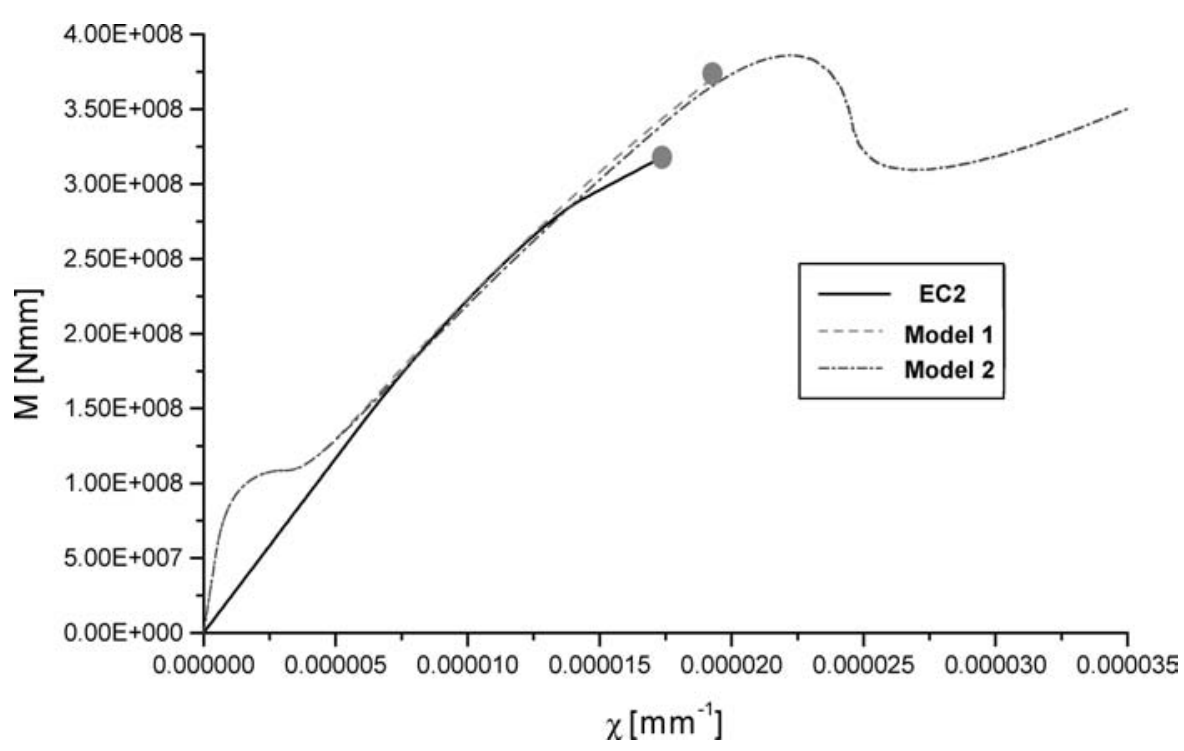

FIG. 10. Bending behavior of the reinforced concrete section obtained considering EC2 mode and the proposed Model 1 and Model 2.

completely damaged in tension, the behavior computed with the three stress-strain laws present only light differences.

In EC2 model and Model 1 the analyses are interrupted when the axial deformation reaches the limit value $\varepsilon_{f}$ in a point of the section, since the compressive concrete behavior is not defined after that point for EC2 model and Model 1, as shown in Figure 9.

In Model 2 plastic and damage evolutions in compression are defined also after the limit deformation $\varepsilon_{f}$. In fact, the softening branch of the response is due to the damage and plastic evolutions.

When the concrete material is completely damaged, only the FRP reinforcements are able to bear bending loading increments; thus, the response becomes linear with a slope corresponding to the bending stiffness of the reinforcements.

\section{CONCLUSIONS}

The proposed one-dimensional model is thermodynamically consistent and it accounts for the damage in tension and for the plasticity and damage in compression. In particular, the plastic evolution is governed by the elastic strain as the experimental evidences show.

The model appears simple and effective. In fact, it is defined by a reduced number of parameters with a clear physical meaning. Moreover, it is able to reproduce different types of mechanical behaviors of the cementitiuous materials properly setting the parameters. In particular, the Eurocode 2 [29] concrete constitutive laws can be reproduced within the proposed model.

The developed numerical procedure, based on the arc-length method, is able to determine the complex behavior of the reinforced cementitiuous elements. The proper choice of the control parameters allows it to follow the load-displacement equilibrium curve, which presents softening and sharp snap-back branches.
The presented one-dimensional model and numerical procedure allow us to derive simple but fundamental considerations on the mechanical response of cementitious elements reinforced by advanced composite materials. In fact, the damage and plastic effects, taken into account in the material constitutive law, significantly influence the cross-section beam behavior. As matter of fact, the results obtained by the presented model could be strongly different from the ones determined adopting more classical and simple constitutive laws, as the elastoplastic or the elastic no tension models. These results can give useful information for a more profitable structural design. In particular, collapse loads and resistance domains of the crosssection in terms of axial force and bending moment can be derived.

Furthermore, the procedure is able to evaluate the behavior of damaged structures before and after the application of reinforcements.

Future developments will deal with the implementation of the elastoplastic-damage model in a finite element code, in the framework of the fiber models, able to overcome the localization problems due to the material softening response.

\section{ACKNOWLEDGMENTS}

The financial supports of the Italian National Research Council (CNR) and of the research network reluis are gratefully acknowledged.

\section{REFERENCES}

1. El-Badry, M., (Editor). Advanced Composite Materials in Bridges and Structures, 2nd International Conference, Montréal, Québec, Canada.

2. Saadatmanesh, H., "Extending service life of concrete and masonry structures with fiber composites," Costruction and Building Materials 11(5-6), 327-335 (1997). 
3. Triantafillou, T. C., "Composites: A new possibility for the shear strengthening of concrete, masonry and wood," Composites Science and Technology 58, 1285-1295 (1998).

4. Redson, J., "Canada's infrastructure benefits from FRP," Reinforced Plastics July/August, 34-38 (1999).

5. Rabinovitch, O., and Frostig, Y., "Nonlinear high-order analysis of cracked RC beams strengthened with FRP strips," Journal of Structural Engineering 127(4), 381-389 (2001).

6. Alagusundaramoorthy, P., Harik, I. E., and Choo, C .C., "Flexural behavior of R/C beams strengthened with carbon fiber reinforced polymer sheets or fabric," Journal of Composites for Construction 7(4), 292-301 (2003).

7. Deniaud, C., and Cheng, J. J. R., "Reinforced concrete T-Beams strengthened in shear with fiber reinforced polymer sheets," Journal of Composites for Construction 7(4), 302-310 (2003).

8. Rabinovitch, O., "Bending behavior of reinforced concrete beams strengthened with composite materials using inelastic and nonlinear adhesives," Journal of Structural Engineering 131(10), 1580-1592.

9. Shao, Y., Aval, S., Mirmiran, A., and M. ASCE. "Fiber-element model for cyclic analysis of concrete-filled fiber reinforced polymer tubes," Journal of Structural Engineering 131(2), 292-303 (2005).

10. Schwegler, G., "Masonry construction strengthened with fiber composites in seismically endangered zones," Proceedings of 10th European Conference on Earthquake Engineering, Rotterdam, 2299-2303.

11. Ehsani, M. R., "Strengthening of earthquake-damaged masonry structures with composite materials" Non-metallic (FRP) Reinforcement for Concrete Structures, L. Taerwe, (Ed.), Chapman \& Hall, London, 680-687 (1995).

12. Laursen, P. T., Seible, F., Hegemier, G. A., and Innamorato, D., "Seismic retrofit and repair of masonry walls with carbon overlays," Non-metallic (FRP) Reinforcement for Concrete Structures. L. Taerwe (Ed.), Chapman \& Hall, London, 616-623.

13. Tumialan, G., Micelli, F., and Nanni, A., Strengthening of Masonry Structures with FRP Composites, Structures 2001, Washington DC, May 21-23, 2001.

14. Luciano, R., Marfia, S., and Sacco, E., Reinforcement of Masonry Arches by FRP Materials: Experimental Tests and Numerical Investigations. Proceedings of Composites in Infrastructure, San Francisco, June 10-12 (2002).

15. Foraboschi, P., "Strengthening of masonry arches with fiber-reinforced polymer strips," Journal of Composites for Construction (C) ASCE, 191-202 (2004).

16. Marfia, S., and Sacco, E., "Numerical procedure for elastoplastic no-tension model," International Journal for Computational Methods in Engineering Science and Mechanics 6, 187-199 (2005).

17. Luciano, R., and Sacco, E., "Damage of masonry panels reinforced by FRP sheets," International Journal of Solids and Structures 35(15), 1723-1741 (1998).

18. Marfia, S., and Sacco, E., "Modeling of reinforced masonry elements," International Journal of Solids and Structures 38, 4177-4198 (2001).

19. Cecchi, A., Milani, G., and Tralli, A., "In-plane loaded CFRP reinforced masonry walls: Mechanical characteristics by homogenisation procedures," Composites Science and Technology 64, 2097-2112 (2004).

20. CNR (NATIONAL RESEARCH COUNCIL) DT 200/2004. Guidelines for Design, Execution and Control of Strengthening Interventions by Means of Fibre-reinforced Composites, CNR, Rome (2004).

21. Wu, Z.J., and Bailey, C. G., "Fracture resistance of a cracked concrete beam post-strengthened with FRP sheets," International Journal of Fracture 135, 35-49 (2005).

22. Ferretti, D., and Savoia, M., "Non-linear model for R/C tensile members strengthened by FRP-plates," Engineering Fracture Mechanics 70, 10691083 (2003).

23. Lee Tai-Kuang, Pan, A. D. E., and Member ASCE., "Analysis of composite beam-columns under lateral cyclic loading," Journal of Structural Engineering 127(2), 186-193 (2001).
24. Aval, S. B. B., Saadeghvaziri, M. A., M. ASCE, and Golafshani A. A., "Comprehensive composite inelastic fiber element for cyclic analysis of concrete-filled steel tube columns," Journal of Engineering Mechanics, 128(4), 428-437 (2002).

25. Marfia, S., Rinaldi, Z., and Sacco, E., "Softening behavior of reinforced concrete beams under cyclic loading," International Journal of Solids and Structures 41, 3293-3316 (2004).

26. Crisfield, M. A., Non-linear Finite Element Analysis of Solids and Structures. Vol. 1. John Wiley \& Sons LTD: England (1991).

27. de Souza Neto, E. A., and Feng, Y. T., "On the determination of the path direction for arc-length methods in the presence of bifurcations and 'snap-backs."' Comput. Methods Appl. Mech. Engrg. 179, 81-89 (1999).

28. Simo, J. C., and Hughes, T. J. R., Computational Inelasticity. SpringerVerlag, New York (1998).

29. Eurocode 2. Design of concrete structures-UNI ENV (1992) -1-1.

30. Meschke, G., Lackner, R., and Mang, H. A., "An anisotropic elastoplasticdamage model for plain concrete," Int. J. Numer. Methods Eng. 42, 703727.

\section{APPENDIX: ANALYTICAL SOLUTION}

An analytical expression for the stress-strain relation $\left(\varepsilon_{o}, \chi\right)-$ $(N, M)$ is obtained. The discussion is herein restricted to the case of rectangular cross-sections with base $b$ and height $h$; the extension to other cross-section geometries can be performed. Two elastic reinforcements are placed one at the bottom and the other at the top of the cross-section, characterized by areas $S_{1}=S$ and $S_{2}=S$ and by abscissas $h_{1}=h / 2$ and $h_{2}=-h / 2$.

The value of the eccentricity $d=M_{e x t} / N_{e x t}$ is set in order to get the whole section subjected only to tensile stress. Hence, only damage and no plasticity can occur in the cohesive beam.

The stress in the cementitious cross-section is given by:

$$
\begin{array}{lll}
\sigma_{e}=E \varepsilon & \text { when } & \varepsilon \leq \varepsilon_{c}^{+} \\
\sigma_{d}=E \varepsilon_{c}^{+} \frac{\left(\varepsilon_{u}^{+}-\varepsilon\right)}{\left(\varepsilon_{u}^{+}-\varepsilon_{c}^{+}\right)} & \text {when } & \varepsilon_{c}^{+}<\varepsilon \leq \varepsilon_{u}^{+} \\
\sigma_{u}=0 & \text { when } & \varepsilon_{u}^{+}<\varepsilon
\end{array}
$$

where the deformation $\varepsilon$ is obtained by formula (21).

Five different cases can occur, depending on the stress distribution in the cross-section, as represented in Figures 1 and 2:

1. the whole section behaves elastically,

2. a part of the section is still elastic and the other part is partially damaged,

3. a part of the section behaves elastically, a part is partially damaged and the rest is completely damaged,

4. a part of the section is partially damaged while the other part is completely damaged,

5. the whole section is completely damage only the elastic reinforcement is able to carry the load increment. 
The two abscissas $y_{c}$ and $y_{u}$ where the initial and the final damage strain thresholds occur, respectively, are defined as:

$$
y_{c}=\frac{\varepsilon_{c}^{+}-e}{\chi} y_{u}=\frac{\varepsilon_{u}^{+}-e}{\chi}
$$

The tensile axial force $N^{B}$ and the bending moment $M^{B}$ in the cohesive beam for the five cases result:

$$
\begin{array}{rlrl}
N_{1}^{B}=\int_{-h / 2}^{h / 2} \sigma_{e} d y & M_{1}^{B}= & \int_{-h / 2}^{h / 2} y \sigma_{e} d y \\
N_{2}^{B}=\int_{-h / 2}^{y_{c}} \sigma_{e} d y+\int_{y_{c}}^{h / 2} \sigma_{d} d y & M_{2}^{B}= & \int_{-h / 2}^{y_{c}} y \sigma_{e} d y \\
& +\int_{y_{c}}^{h / 2} y \sigma_{d} d y \\
N_{3}^{B}=\int_{-h / 2}^{y_{c}} \sigma_{e} d y+\int_{y_{u}}^{y_{c}} \sigma_{d} d y & M_{3}^{B}= & \int_{-h / 2}^{y_{c}} y \sigma_{e} d y \\
& & & +\int_{y_{c}}^{y_{u}} y \sigma_{d} d y \\
N_{4}^{B}=\int_{h / 2}^{y_{u}} \sigma_{d} d y & M_{4}^{B}= & \int_{-h / 2}^{y_{u}} y \sigma d y \\
N_{5}^{B}=0 & M_{5}^{B}= & 0
\end{array}
$$

The axial force $N^{R}$ and the bending moment $M^{R}$ in the elastic reinforcements are:

$$
\begin{aligned}
& N^{R}=S E^{R}\left[\varepsilon\left(\frac{h}{2}\right)+\varepsilon\left(-\frac{h}{2}\right)\right]=2 S E^{R} e \\
& M^{R}=\frac{h}{2} S E^{R}\left[\varepsilon\left(\frac{h}{2}\right)-\varepsilon\left(-\frac{h}{2}\right)\right]=\frac{h^{2}}{2} S E^{R} \chi
\end{aligned}
$$

For the $i$-th case, with $i=1, \ldots, 5$, the total axial force and bending moment acting in the cross-section result:

$$
\begin{aligned}
N_{i}^{t o t} & =N_{i}^{B}+N^{R} \\
M_{i}^{t o t} & =M_{i}^{B}+M^{R}
\end{aligned}
$$

During the loading history, it is set:

$$
M_{i}^{t o t}=N_{i}^{t o t} d
$$

From Eq. (59) the relations $\chi(e)$ or $e(\chi)$ are obtained. Substituting $\chi(e)$ in the Eq. (57), the relationship $N_{i}^{\text {tot }}-e$ is determined for all the five different cases. Then, substituting $e(\chi)$ in Eq. (58), the relation $M_{i}^{\text {tot }}-\chi$ is obtained for all the five different cases. 
Copyright of Mechanics of Advanced Materials \& Structures is the property of Taylor \& Francis Ltd and its content may not be copied or emailed to multiple sites or posted to a listserv without the copyright holder's express written permission. However, users may print, download, or email articles for individual use. 